\title{
Recommendations for PDF usage in LHC predictions
}

\section{Ringailè Plačakyte்}

Universität Hamburg, II. Institut für Theoretische Physik

E-mail: ringaile@mail.desy.de

\begin{abstract}
A short review of the currently available modern parton distribution functions (PDFs) and the theory predictions obtained using those PDFs for several benchmark processes at LHC, including Higgs boson production, is presented in this write-up. It includes the discussion on theory assumptions made in the determination procedure of PDFs and an impact on the differences in the obtained predictions, followed by the alternative to PDF4LHC recommendations for the usage of PDF sets for theory predictions at the LHC.
\end{abstract}

XXIV International Workshop on Deep-Inelastic Scattering and Related Subjects

11-15 April, 2016

DESY Hamburg, Germany

\footnotetext{
* Speaker.
} 


\section{Overview of modern PDFs}

The wealth of precise measurements from the Large Hadron Collider (LHC) including the new data at the highest center-of-mass collision energies ever achieved $(\sqrt{s}=13 \mathrm{TeV})$ are used to search for physics phenomena beyond the SM (BSM). The measurements are compared to precise theoretical predictions, which, ideally, incorporate higher order radiative corrections in Quantum Chromodynamics (QCD) and the electroweak sector of the SM. Parton distribution functions (PDFs) in the proton are an essential input for any cross section prediction at hadron colliders. Despite steady improvements in the accuracy of PDF determinations in the last years, the uncertainties associated with PDFs, likewise and the strong coupling $\alpha_{s}\left(M_{Z}\right)$ and heavy quark masses, still dominate many calculations of cross sections for SM processes at the LHC. The currently available PDF sets are CJ15 [1], accurate to NLO in QCD, as well as ABM12 [2], CT14 [3], HERAPDF2.0 [4], JR14 [5], MMHT14 [6], and NNPDF3.0 [7] to NNLO in QCD. Both theoretical and experimental inputs used in the PDF analyses have direct impact on the obtained nonperturbative parameters, namely, the fitted PDFs, the value of $\alpha_{s}\left(M_{Z}\right)$ and the quark masses. For precision predictions in Run 2 of the LHC it is therefore very important to quantify those effects in order to reduce the uncertainties in the nonperturbative input parameters. The benchmark of individual PDFs, including the combined sets as proposed in the recent PDF4LHC recommendations [8], is performed for various processes at hadron colliders and presented together with the detailed discussion on the underlying theory assumptions in the PDF analysis procedure, followed by the alternative recommendations for the usage of PDFs for theory predictions at the LHC.

\section{Cross section predictions for the LHC}

The detailed overview of the currently available data which can be used to determine PDFs together with the PDF fit results from different groups is provided in the original study in Ref. [9]. The basic theoretical issues for a consistent determination of the PDFs from DIS and other hard scattering data is described in the same reference. In following, few selected examples are discussed, namely the value of the strong coupling constant, Higgs cross section predictions at LHC, hadro-production of heavy quarks and heavy $W^{\prime}$ boson production.

\subsection{The strong coupling constant}

The value of the strong coupling constant $\alpha_{s}\left(M_{Z}\right)$ is one of the most important parameters in QCD which has a direct impact on the size of a number of cross sections at the LHC such as Higgs production. In the context of global PDF analyses $\alpha_{s}\left(M_{Z}\right)$ has a particular interest due to its correlation with the gluon PDF and the charm-quark mass. The value of $\alpha_{s}\left(M_{Z}\right)$ is determined from a large number of different processes and methods at different scales and yields the world average $0.1181 \pm 0.0013$ at NNLO [10]. While the world average of $\alpha_{s}\left(M_{Z}\right)$ is closely related to the values determined in PDF fits, two important aspects specific to PDF analyses are addressed here: First, in some PDF analyses $\alpha_{s}\left(M_{Z}\right)$ is treated as a free parameter which allows to control its correlation with other PDF parameters. Second, a large spread of $\alpha_{s}\left(M_{Z}\right)$ values are used (or determined) in PDFs, ranging from 0.1132 to 0.1183 , see Table 1 . These differences can be traced back to different data sets used or to different theory assumptions applied, as indicated in Tab 1. 
One example is the data for the hadro-production of jets at LHC where full higher than NLO order corrections are not yet available but are known to be significant - various PDF groups employ different methods to account for this inconsistency in NNLO fits which in own turn, may lead to different $\alpha_{s}\left(M_{Z}\right)$ values. Examples of different methodology in PDF fits which are potential sources of discrepancies in $\alpha_{s}\left(M_{Z}\right)$ values are nuclear corrections, heavy-flavour schemes, etc. as discussed in Ref. [9]. In addition, the bias also may be introduced by fixing $\alpha_{s}\left(M_{Z}\right)$ to the same value at different orders (NLO and NNLO) in PDF sets used in the combination procedure of PDF4LHC recommendations [8], which is in contradiction with the precision determinations of $\alpha_{s}\left(M_{Z}\right)$ at different orders in perturbation theory.

\begin{tabular}{|c|c|c|}
\hline \hline ABM12 [2] & $0.11320 \pm 0.0011$ & fit at NNLO \\
\hline CJ15 [1] & $0.11830 \pm 0.0002$ & fit at NLO \\
\hline CT14 [3] & 0.118 & assumed at NNLO \\
\hline HERAPDF2.0Jets [4] & $0.1183+0.0040-0.0034$ & fit at NLO \\
\hline JR14 [5] & $0.11360 \pm 0.0004$ & dynamical fit at NNLO \\
& $0.11620 \pm 0.0006$ & standard fit at NNLO \\
\hline MMHT14 [9] & 0.118 & assumed at NNLO \\
\hline NNPDF3.0 [7] & $0.115-0.121$ & assumed at NNLO; preferred value 0.118 \\
\hline PDF4LHC15 [8] & 0.118 & assumed at NNLO \\
\hline \hline
\end{tabular}

Table 1: Values of $\alpha_{s}\left(M_{Z}\right)$ obtained or used in the nominal PDF sets of the various groups.

\subsection{Higgs cross section predictions at LHC}

The dominant production mechanism for the SM Higgs boson at the LHC is the gluon-gluon fusion process which is known to $\mathrm{N}^{3} \mathrm{LO}$ in QCD $[11,12]$. Currently the largest source of uncertainties in the predictions of the Higgs cross section are the value of $\alpha_{s}$ and the PDFs.

A large spread for Higgs predictions observed from different PDFs with a range $38.0-42.6 \mathrm{pb}$ using the nominal value of $\alpha_{s}\left(M_{Z}\right)(11 \%)$ and $39.0-44.7 \mathrm{pb}$ if a fixed value of $\alpha_{s}\left(M_{Z}\right)=0.115-$ $0.118(13 \%)$ is used (for details please see the original study [9]). This illustrates the importance of controlling the accuracy and the correlation of the strong coupling constant with the PDF parameters and the heavy quark masses in fits. As an illustration, Tab. 2 shows the values of the charmquark mass $m_{c}^{\text {pole }}, \alpha_{s}\left(M_{Z}\right)$ and $\chi^{2}$ value obtained using the open-source package xFitter [16] for the HERA charm data [13] together with the calculated Higgs cross section at NNLO, using MSTW PDFs. Here, a linear rise of the cross section for increasing values of charm mass is observed (3\% for the best fit $\alpha_{s}\left(M_{Z}\right)$ in the range from 1.15 to $1.55 \mathrm{GeV}$ and $1 \%$ for fixed). The latter case leads to a best fit of $m_{c}^{\text {pole }}=1.2 \mathrm{GeV}$ which is significantly smaller than the nominal fit with $m_{c}^{\text {pole }}=1.4 \mathrm{GeV}$. These values are not compatible with the world average of the PDG [14] and indicate that the charm-quark mass effectively takes over the role of a 'tuning' parameter. Similar results have been observed in other PDF analyses (please see original study [9]). These results are in agreement with the results shown in e.g. CT10 study [10], where about $2 \%$ difference in Higgs cross section is observed for $m_{c}^{\text {pole }}$ changing in the range from 1.0 to $1.36 \mathrm{GeV}$ (please note the smaller value of the $m_{c}^{\text {pole }}$ range than the one considered above).

Finally, for the calculation of PDF uncertainties in precision observables (such as the Higgs cross section), the combined PDF set is recommended to use in PDF4LHC recommendations [8]. It is 


\begin{tabular}{|c|c|c|c|c|}
\hline \hline$m_{c}^{\text {pole }}$ & $\alpha_{s}\left(M_{Z}\right)$ & $\chi^{2} / N D P$ & $\sigma(H)^{N N L O}[p b]$ best fit $\alpha_{s}\left(M_{Z}\right)$ & $\sigma(H)^{N N L O}[p b] \alpha_{s}\left(M_{Z}\right)=0.118$ \\
\hline 1.15 & 0.1164 & $78 / 52(71 / 52)$ & 41.01 & $(42.05)$ \\
\hline 1.2 & 0.1166 & $76 / 52(70 / 52)$ & 41.18 & $(42.11)$ \\
\hline 1.25 & 0.1167 & $75 / 52(76 / 52)$ & 41.33 & $(42.17)$ \\
\hline 1.3 & 0.1169 & $76 / 52(77 / 52)$ & 41.48 & $(42.25)$ \\
\hline 1.35 & 0.1171 & $78 / 52(79 / 52)$ & 41.68 & $(42.30)$ \\
\hline 1.4 & 0.1172 & $82 / 52(83 / 52)$ & 41.83 & $(42.45)$ \\
\hline 1.45 & 0.1173 & $88 / 52(89 / 52)$ & 42.00 & $(42.51)$ \\
\hline 1.5 & 0.1173 & $96 / 52(96 / 52)$ & 42.14 & $(42.58)$ \\
\hline 1.55 & 0.1175 & $105 / 52(106 / 52)$ & 42.29 & \\
\hline \hline
\end{tabular}

Table 2: The values of the charm-quark mass and $\alpha_{s}\left(M_{Z}\right)$ in MMHT analysis [9] together with the value for $\chi^{2} / N D P$ for the HERA data [13] and the Higgs cross section at NNLO at $\sqrt{s}=13 \mathrm{TeV}$ for $m_{H}=125 \mathrm{GeV}$ at the nominal scale $m_{H}$. The numbers in parentheses are obtained with the value of $\alpha_{S}\left(M_{Z}\right)$ fixed to 0.118

unclear why PDFs should be treated differently in this case than in the case of comparisons between data and theory for SM measurements (point 1 of recommendations), since these differences are smeared out in the combination and, in addition, the comparison is limited to only three PDF sets.

\subsection{Hadro-production of heavy quarks}

Charm-quark hadro-production provides the possibility to check the consistency of different PDFs. The inclusive cross section of the reaction $p p \rightarrow c \bar{c}$ compared with theory predictions at NLO and NNLO using various PDFs as a function of the center-of mass energy $\sqrt{s}$ to available experimental data is shown in Fig. 1. All predictions agree well with data at low energies but start to behave differently for HERAPDF2.0, MMHT14 or NNPDF3.0 at energies above $\sqrt{s} \simeq \mathscr{O}(10)$ $\mathrm{TeV}$. Despite that at these energies the associated PDF uncertainties become very large, the striking feature seen in Fig. 1 is the negative cross sections for HERAPDF2.0, MMHT14 and PDF4LHC15 at NNLO. This feature is caused by the negative gluon in the NNLO fit of those PDFs illustrating an instability of the perturbative expansion of the inclusive $p p \rightarrow c \bar{c}$ cross sections at energies when the contribution from the quark-gluon channel dominates.

\subsection{Heavy $W^{\prime}$ boson}

The PDFs at large- $x$ are not known precisely due to limited available experimental data and due to various approximations in the treatment of nuclear corrections for deuterium data, target mass and higher twist effects. This can be illustrated with the production of a heavy $W^{\prime}$ boson as a function of the $W^{\prime}$ rapidity, $y_{W^{\prime}}$. Fig. 2 shows the uncertainty in the parton luminosity for a produced negatively charged $W^{\prime}$ boson for various PDF sets. Here a very large range of uncertainties is observed for the various PDF sets due to different tolerance criteria and methodologies used for the treatment of data at high values of $x$. The smallest uncertainty is obtained for the CJ15 PDF set, which makes use of low invariant mass data to constrain the high- $x$ region, and does not employ additional tolerance factors inflating the uncertainties. The MMHT and CT14 PDF sets have larger errors, due to stronger cuts on low-mass DIS data and larger tolerances, and consequently the combined PDF4LHC15 set gives similarly large uncertainties. This example illustrates the problematic 

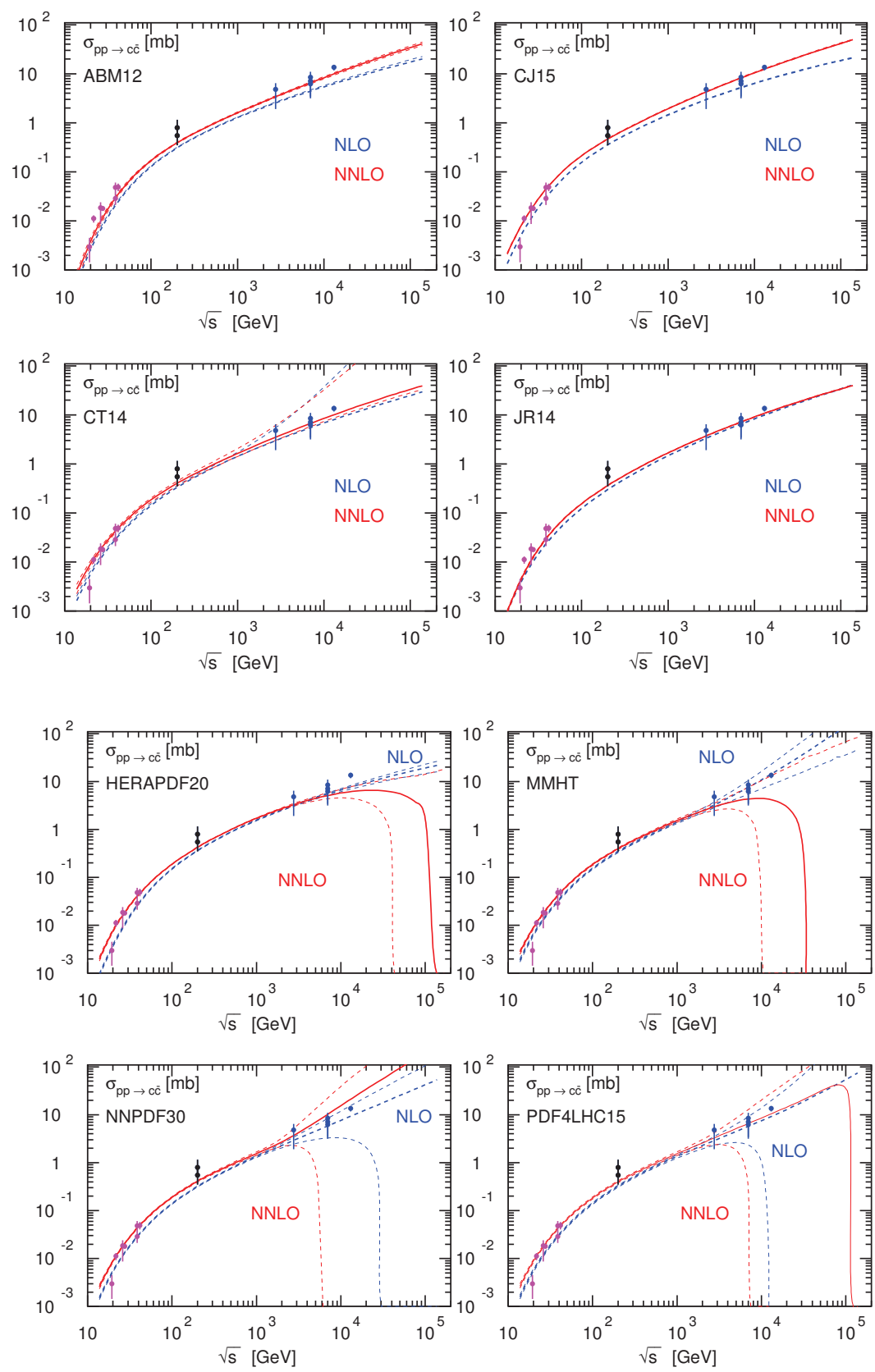

Figure 1: Theoretical predictions for the total $p p \rightarrow c \bar{c}$ cross section as a function of the center-of-mass energy $\sqrt{s}$ at NLO (dashed lines) and NNLO (solid lines) in the $\bar{M} S$ scheme with $m_{c}\left(m_{c}\right)=1.27 \mathrm{GeV}$ and scale choice $\mu_{R}=\mu_{F}=2 m_{c}\left(m_{c}\right)$ using various PDF sets (PDF uncertainties shown as dashed lines)

nature of statistically combined PDF sets that have been determined using very different theoretical treatments of the high- $x$ region, leading to a possible overestimate of the uncertainties at these kinematics. 


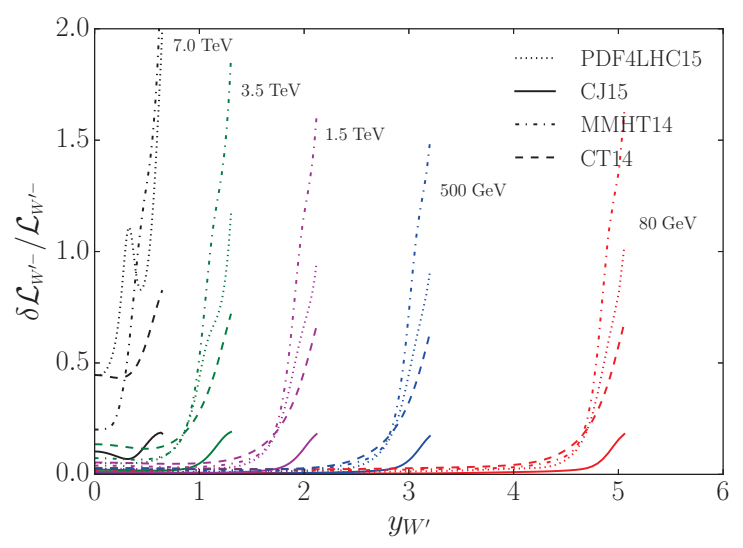

Figure 2: Relative uncertainty $\delta \mathscr{L}_{W^{\prime}} / \mathscr{L}_{W^{\prime-}}$ in the $W^{\prime-}$ luminosity as a function of rapidity $y_{W^{\prime}}$ for the combined PDF4LHC15 set (dotted), the CJ15 (solid), MMHT14 (dotdashed), and CT14 (dashed) PDFs for various $W^{\prime}$ masses. All PDF uncertainties have been scaled to a common $68 \%$ c.l.

\section{Alternative recommendations for PDF usage}

Based on the presented benchmark results and the aspects discussed regarding the official PDF4LHC recommendations [8], we propose modifications to the recommendations for PDF usage at the LHC which allow to retain the predictive capability of the individual PDF sets. Two cases can be distinguished:

1 Precise theory predictions: Recommendation: Use the individual recent PDF sets, currently ABM12 [2], CJ15 [1], CT14 [3], JR14 [5], HERAPDF2.0 [4], MMHT14 [6], and NNPDF3.0 [7] (or as many as possible), together with the respective uncertainties for the chosen PDF set, the strong coupling $\alpha_{s}\left(M_{Z}\right)$ and the heavy quark masses $m_{c}, m_{b}$ and $m_{t}$. Once a PDF set is updated, the most recent version should be used.

2 Theory predictions for feasibility studies: Use any of the recent PDF sets.

\section{Outlook}

Several aspects in PDF extraction have been briefly discussed here, which emphasize the importance of the selection of consistent data sets and of the different theoretical assumptions in PDF fits. The main thrust of the study has been the computation of benchmark cross sections for a variety of processes at hadron colliders, including Higgs boson production in gluon-gluon fusion. It has been illustrated how different choices for the theoretical description of the hard scattering process and choices of parameters have an impact on the predicted cross sections, and lead to systematic shifts that are often significantly larger than the associated PDF and $\alpha_{s}\left(M_{Z}\right)$ uncertainties. We aim at bringing the points considered and shortcomings exposed in the recent PDF4LHC recommendations [8] into discussions and to propose alternative recommendations for the PDF usage. Ideally, as a consequence of these discussions, the next recommendations would include these observations and would be published as a common work of the all PDF groups.

Acknowledgments I would like to thank authors of Ref. [9], A. Accardi, S. Alekhin, J. Blümlein, M.V. Garzelli, K. Lipka, W. Melnitchouk, S. Moch, J.F. Owens, E. Reya, N. Sato, A. Vogt and $\mathrm{O}$. Zenaiev for their help in preparing the write-up. 


\section{References}

[1] A. Accardi, L. T. Brady, W. Melnitchouk, J. F. Owens, and N. Sato, Constraints on large-x parton distributions from new weak boson production and deep-inelastic scattering data, Phys. Rev. D93 (2016) 114017, [arXiv:1602.03154]

[2] S. Alekhin, J. Blümlein, and S. Moch, The ABM parton distributions tuned to LHC data, Phys. Rev. D89 (2014) 054028, [arXiv:1310.3059]

[3] S. Dulat, T. J. Hou, J. Gao, M. Guzzi, J. Huston, P. Nadolsky, J. Pumplin, C. Schmidt, D. Stump, and C. P. Yuan, The CT14 global analysis of Quantum Chromodynamics, [arXiv:1506.07443]

[4] H1 and ZEUS Collaborations, H. Abramowicz et al., Combination of measurements of inclusive deep-inelastic ep scattering cross sections and QCD analysis of HERA data, [arXiv:1506.06042]

[5] P. Jimenez-Delgado and E. Reya, Delineating parton distributions and the strong coupling, Phys. Rev. D89 (2014) 074049, [arXiv:1403.1852]

[6] L. A. Harland-Lang, A. D. Martin, P. Motylinski, and R. S. Thorne, Parton distributions in the LHC era: MMHT 2014 PDFs, Eur. Phys. J. C75 (2015) 204, [arXiv:1412.3989]

[7] NNPDF Collaboration, R. D. Ball et al., Parton distributions for the LHC Run II, JHEP 04 (2015) 040, [arXiv:1410.8849]

[8] J. Butterworth et al., PDF4LHC recommendations for LHC Run II, J. Phys. G43 (2016) 023001, [arXiv:1510.03865]

[9] A. Accardi et al. A Critical Appraisal and Evaluation of Modern PDFs, DESY-16-041, DO-TH-16-05, JLAB-THY-16-2231, LTH-1081, Eur. Phys. J. C76 (2016) no.8, 471, [arXiv:1603.08906]

[10] Particle Data Group Collaboration, K. A. Olive et al., Review of particle physics, Chin. Phys. C38 (2014) 090001

[11] L. A. Harland-Lang, A. D. Martin, P. Motylinski, and R. S. Thorne, Charm and beauty quark masses in the MMHT2014 global PDF analysis, [arXiv:1510.02332]

[12] C. Anastasiou, C. Duhr, F. Dulat, E. Furlan, T. Gehrmann, F. Herzog, A. Lazopoulos, and B. Mistlberger, High precision determination of the gluon fusion Higgs boson cross section at the LHC, [arXiv:1602.00695]

[13] C. Anastasiou, C. Duhr, F. Dulat, F. Herzog, and B. Mistlberger, Higgs boson gluon fusion production in QCD at three loops, Phys. Rev. Lett. 114 (2015) 212001, [arXiv:1503.06056]

[14] H1 and ZEUS Collaborations, H. Abramowicz et al., Combination and QCD analysis of charm production cross section measurements in deep-inelastic ep scattering at HERA, Eur. Phys. J. C73 (2013) 2311, [arXiv:1211.1182]

[15] J. Gao, M. Guzzi, and P. M. Nadolsky, Charm quark mass dependence in a global QCD analysis, Eur.Phys.J. C73 (2013) 2541, [arXiv:1304.3494]

[16] S. Alekhin, O. Behnke, P. Belov, S. Borroni, M. Botje, et al., HERAFitter, Open source QCD fit project, Eur.Phys.J. C75 (2015) 304, [arXiv:1410.4412] and http://xFitter.org 